\title{
Hacia una nueva edición crítica de las Alegorías de la Ilíada de Tzetzes (I): ¿Dos versiones Tzetzianas?
}

\author{
Carlos Martins de Jesus ${ }^{1}$
}

Recibido: 11 de Agosto de 2019 / Aceptado: 23 de Septiembre de 2019

\begin{abstract}
Resumen. Escritas entre 1143 y 1146 bajo el patrocinio de Berta de Sulzbach, la que pronto sería la Emperatriz Irene, esposa de Manuel I Comneno, las Alegorías de la Ilíada son un resumen, una paráfrasis y especialmente una explicación alegórica, en más de 6.600 versos políticos, de los elementos sobrenaturales paganos del poema de Homero. Las Alegorías de Tzetzes han recibido poca atención, y solamente en los últimos años los críticos han fijado su atención en ellas. A otro nivel, el poema carece de una edición crítica actualizada, ya que no se edita desde mediados del siglo XIX (Matranga 1850; Boissonade 1851). Hasta el momento, he localizado un total de 23 códices jamás colacionados y que merecen serlo, de los 41 que lista la web de Pinakes: nueve recensiones completas y otras 14 parciales, en dos casos solamente entre los scholia a la Ilíada de Homero. Crucial parece ser el hecho de que los dos testimonios manuscritos más antiguos, el Barocci 131, ff. 42-62v (D) y el Vindob. phil. gr. 305, ff. 1-145v (E), se cuenten entre esos códices no considerados. El propósito de este artículo es presentar las razones por las que una nueva edición crítica es necesaria, analizando algunos de los códices jamás utilizados para editar el poema, a la vez que busca formular algunas conclusiones previas sobre ellos, especialmente la posibilidad de que el propio Tzetzes hubiese preparado dos versiones de su obra.

Palabras clave: Juan Tzetzes; Homero; Alegorías de la Ilíada; edición crítica.
\end{abstract}

\section{[en] Towards a new critical edition of Tzetzes' Allegories of the Iliad (I): Two Tzetzian versions?}

Abstract. Written in between 1143 and 1146 under the commission of Bertha von Sulzbach, soon to be the Empress Eirene, wife of Manuel I Komenos, the Allegories of the Iliad are an over 6.600 political-verse summary, paraphrase, and mostly allegorical explanation of the pagan supernatural elements of Homer's poem. Tzetzes' Allegories have received little attention, and only in the past years did scholars focused on them. Furthermore, the poem still lacks a up-to-date edition, as it has not been edited since the mid-nineteenth century (Matranga 1850; Boissonade 1851). So-far, I came to a total of 23 valuable uncollated codices, out of the 41 listed by the Pinakes website: nine full recensions and 14 partial recensions, in two cases among the scholia to Homer's Iliad. Meaningful is the fact that the two oldest manuscripts, the Barocci 131, ff. 42-62v (D) and Vindob. phil. gr. 305, ff. 1-145v (E) are among those codices never taken in consideration. The aim of this paper is to present the reasons for which a new edition is needed, by looking at the poem's several unconsidered manuscript sources and trying to elaborate some previous conclusions on them, namely by exploring the possibility of Tzetzes' preparation of two versions of his work.

Keywords: John Tzetzes; Homer; Allegories of the Iliad; critical edition.

Cómo citar: Martins de Jesus, C. (2020), Hacia una nueva edición crítica de las Alegorías de la Ilíada de Tzetzes (I): ¿Dos versiones Tzetzianas?, en Cuadernos de Filología Clásica. Estudios griegos e indoeuropeos 30, 189-197.

\footnotetext{
1 Universidade de Coimbra carlos.jesus@uc.pt
} 
Juan Tzetzes ( $c a .1110-1180$ ), ejemplo máximo del clasicista profesional del período medio-bizantino, fue un erudito que vivió del oficio del magisterio y de rescribir y comentar, bajo patrocinio aristocrático, un conjunto de autores griegos clásicos, con un papel especialmente destacado para Homero, habiendo producido una obra a la que, no hace mucho tiempo, Kaldellis (2007: 301) llamó "classics for dummies". Dado que nos parece una expresión injustamente despectiva, preferiríamos sustituirla por "clásicos para iniciados", con Homero y sus dos poemas épicos a la cabeza, dando continuidad a una larguísima tradición de rescritura y reinterpretación de sus dos poemas épicos, muy destacada en toda la literatura bizantina ${ }^{2}$. A ellos, en efecto, dedicó Tzetzes gran parte de su carrera ${ }^{3}$, componiendo los Carmina Iliaca (ca. 1133), reescritura de los sucesos de la guerra de Troya, sus antecedentes y consecuencias - la obra, en hexámetros, se divide en tres secciones, Antehomerica, Homerica y Posthomerica -, una larguísima Exegesis de la Ilíada (ca. 1140), y las Alegorías de la Ilíada y de la Odisea (ca. 1158).

Las Alegorías de la Ilíada que aquí nos importan empezaron por ser un encargo de Berta von Sulzbach, la princesa de Baviera que acababa de llegar a Constantinopla para convertirse, en 1142, en la esposa de Manuel I Comneno ${ }^{4}$. Para ella, para que se enterase de los mitos y significados de una obra que a menudo era asunto de conversación en los eventos que empezaba a frecuentar, Tzetzes compuso los primeros 15 libros del poema. Por motivos que desconocemos, la misión fue interrumpida, y solamente pudo concluir los libros 16-24 bajo el patrocinio de un nuevo mecenas, Constantino Cotortzes, para quien escribe una nueva (y muy sencilla) dedicatoria, en la apertura del libro 16 (vv. 1-7).

El poema, con sus 6.600 versos de quince sílabas (el denominado verso político bizantino), mezcla partes que recompendian la Ilíada con otras que son una paráfrasis y sobre todo una explicación alegórica de sus elementos paganos y sobrenaturales, dando como resultado una obra frecuentemente repetitiva y algo barroca, razón por la que, tal vez, no ha recibido mucha atención de parte de los eruditos y filólogos dedicados al mundo bizantino. De hecho, solo en los últimos años se le han dedicado algunos trabajos, en concreto sobre el método alegórico empleado ${ }^{5}$, además de otros que estudian la obra en el marco más amplio de la recepción del texto y los temas homéricos en la literatura bizantina ${ }^{6}$.

Igual infortunio crítico conoció su status editionum, el asunto de este trabajo. Por oposición a lo que pasa con los Carmina Iliaca e incluso las Alegorías de la Odisea ${ }^{8}$, las Alegorías de la Ilíada no conocen una edición crítica desde mediados del siglo XIX, cuando, con tan solo un año de intervalo, las editaron Matranga (1850: 1-223) y Boissonade (1851: 1-340). La obra conoció, hace pocos años, una edición bilingüe griego-inglés (Goldwyn \& Kokkini 2015). Esta, aparte del mérito de publicar por primera vez una traducción del poema en una lengua moderna, con relación al texto griego se limita a reimprimir la edición de Boissonade, haciendo "correcciones tácitas en la puntuación, acentuación y capitalización” y corrigiendo “algunas faltas tipográficas" (Goldwyn \& Kokkini 2015: 517).

La edición de Boissonade, la más reciente, se basa en tan solo tres manuscritos parisienses (Par. gr. 2707, ca. 1300/1301 [A]; Par. gr. 2705 [B] y 2644 [C], ambos del siglo XIV). En cuanto a la edición de Matranga, ya conocida y citada por Bois-

Browning (1975 y 1992), Matzukis (1992).

Browning (1975: 26-28), Morgan (1983), Budelmann (2002: 141-169), Kaldellis (2007: 301-307), Braccini (2011).

Hill (1999: 171-173), Rhoby (2010: 160).

Cesaretti (1991: 134-137), Goldwin (2017).

Budelmann (2002: 141-70), Kaldellis (2007: 301-307 y 2009: 26-29).

Ed. Leone (1995).

Ed. Hunger (1955 y 1956). 
sonade, además de editar los escolios del proprio Tzetzes a su poema, identifica en las páginas 709-748 las variantes de seis manuscritos de la Biblioteca del Vaticano (Pal. gr. 316 [a], del siglo XV; Ottob. gr. 324 [b], del XIV-XV; Vat. gr. 1369 [c], ¿del XIV?; Vat. gr. 904 [d], del XIII-XIV; Vat. gr. 1405 [e], del XV; Vat. gr. 1759 [f], del $\mathrm{XV}-\mathrm{XVII})^{9}$. Además, hecho no menos importante, edita un mayor número de versos en muchos cantos del poema, por lo que, como apunta Mastronarde (2015), Goldwyn \& Kokkini 2015 deberían, como mínimo, haber confrontado ambas ediciones y completado la de Boissonade con las líneas editadas por Matranga que le faltan.

Pero dicha tarea no hubiese sido suficiente, lo dice Mastronarde y lo repetimos nosotros, para cumplir las necesidades editoriales de las Alegorías. Una búsqueda en la web de Pinakes ha dado como resultado que el poema es trasmitido, total o parcialmente, por 41 códices. Eliminadas las repeticiones y los casos en los que apenas un número reducido de versos es copiado (por ejemplo, en el contexto de escolios a los poemas homéricos), llegamos a un total de 23 manuscritos, entre los siglos XIII y XVII, que jamás han sido colacionados por ninguno de los dos editores mencionados con anterioridad. De ellos, en un primer momento, nos centramos en las recensiones completas del poema, las cuales presentamos en un primer intento de ordenación cronológica basado exclusivamente en las descripciones de los catálogos y alguna que otra evidencia externa:

(1) [D] Barocci 131, ff. 42-62v. Biblioteca Bodleiana. Oxford. 1250-1280.

(2) [E] Vindob. phil. gr. 305, ff. 1-145v. Österreichische Nationalbibliothek. 1280.

(3) [F] Barb. gr. 30, ff. 56v-112v. Biblioteca Apostolica Vaticana. Siglo XIII.

(4) [G] Barocci 24, ff. 1r-199r. Biblioteca Bodleiana. Oxford. Siglos XIII/XIV.

(5) [H] Vindob. phil. gr. 118, ff. 1-149v. Österreichische Nationalbibliothek. Siglo XIV.

(6) [I] Ambr. gr. H 27 inf., ff. 052-*. Biblioteca Ambrosiana. Siglo XIV.

(7) [J] Escor. gr. Y.III.20, ff. 1-133. Real Biblioteca de El Escorial. Siglo XV.

(8) [K] Escor. gr. R.III.15, ff. 3-158. Real Biblioteca de El Escorial. Siglo XVI. [Escor. Boiss.]

(9) [L] Auct. T. 1. 10 [Misc. 188], ff. 307-402. Biblioteca Bodleiana. Oxford. Siglo XVI.

La necesidad de una nueva edición crítica resulta evidente, simplemente por el hecho de que los dos primeros códices del listado anterior (Barocci 131 ${ }^{10}$, ff. 42-62v. [D] y Vindob. phil. gr. $305^{11}$, ff. 1-145v. [E] $)^{12}$ tienen fuertes posibilidades de ser los dos testimonios textuales más antiguos del poema, ninguno de los cuales ha sido colacionado ni por Matranga ni por Boissonade. Igual suerte han corrido $\mathbf{F}$ y $\mathbf{G}$, quienes, pudiendo también pertenecer al siglo XIII, tienen no obstante una datación más problemática, por lo que su estudio queda aplazado de momento. En efecto, el códice más antiguo del aparato de Boissonade (el Par. gr. 2707 [A]) no es anterior a 1300/1301 (de 20 a 50 años posterior a nuestros $\mathbf{D}$ y $\mathbf{E}$ ); asimismo, entre los manuscritos colacionados por

9 Matranga tiene constancia de un número más grande de manuscritos que transmiten la obra, confesando que su edición apenas contempla algunos de los códices disponibles: "Quum nulla fere sit mss. bibliotheca, in qua Tzetzae allegoriarum partes non asserventur, Vaticanae quamplurimi codices, ut integrum opus evadat, commodam mihi praestitere utilitatem" (Matranga 1850: 9).

10 Vid. Lámina 1. Catálogo: Coxe (1883, repr. 1969). Disponible online (último acceso 10-08-2019): https://medieval.bodleian.ox.ac.uk/catalog/manuscript_957.

11 Vid. Lámina 2. Manuscrito, papel. Catálogo: Hunger (1961).

12 Las siglas que utilizamos para referirnos a los manuscritos que por primera vez se colacionan retoman las utilizadas por Boissonade (1851) para los tres únicos manuscritos que considera. 
Matranga, apenas d (Vat. gr. 904, ff. 12-62v) puede pertenecer aún al siglo XIII, pero su datación no es precisa, pudiendo haber sido copiado ya en el siglo XIV.

El propósito de este trabajo, tan solo el comienzo de un proceso de recensio que promete ser extenso y complejo, es ensayar una explicación para la constatación de Mastronarde (2015), que en su reseña de la edición de Goldwyn \& Kokkini (2015) identifica un conjunto de versos editados por Matranga (1850) y no por Boissonade (1851), en concreto los que se presentan a continuación:

Prooem. 534-535, 642, 720

9.103-104

11.60, 67-68, 77-81, 97-118, 133-135, 158-159, 164-166, 174-175, 183-190, 224234

12. $28,34,80-84,152,162-172$

13.64-66, 76-79

15.213-215

16.190, 198-199

18.447

20.172-173, 335-336

21.91, 270-271

24.11

Asimismo, hemos buscado en los códices más antiguos aún por colacionar (D y E) argumentos que sostengan una de dos posibilidades planteadas por el mismo Mastronarde: (1) que el proprio Tzetzes haya modificado la obra a lo largo de su vida, y que por lo tanto haya que considerar la existencia de dos versiones distintas, ambas de su responsabilidad; o (2) que la versión más corta - la que imprime Boissonade - sea resultado de supresiones posteriores. Los códices D y E copian, en el cuerpo principal o al margen algunos de estos versos que formarían parte de la versión más larga (y no solamente los apuntados por Mastronarde), prueba de que sus copistas disponían, a la distancia de 100-130 años de la última composición de Tzetzes, o bien de dos versiones distintas, o bien de uno o más códices que copiaban ya estas líneas al margen. Dicho de otra manera, $\mathbf{D}$ y $\mathbf{E}$ forman parte de una tradición que ya considera espurios o menos importantes algunos versos versos, ya sea por influencia directa de otros códices, o bien por confrontación de dos modelos distintos. De ser así, dos posibilidades se abren para el entendimiento de la transmisión primordial del poema, que podemos sintetizar en el árbol que presentamos a continuación:

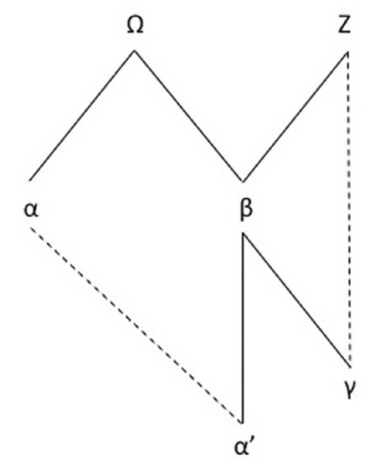


$\boldsymbol{\Omega}$, la versión completa del poema, puede haber dado origen a dos arquetipos, uno que la copia por completo $(\boldsymbol{\alpha})$, y otro que por alguna razón le amputa determinados versos para suprimirlos o copiarlos al margen $(\boldsymbol{\beta})$. Con respecto a $\gamma$, arquetipo de los códices que copian la versión más corta del texto (la tradición textual imprimida por Boissonade), puede o bien haber surgido de $\boldsymbol{\beta}$, por eliminación de dichos versos, o incluso directamente de esa otra tradición textual más corta, de la que habría sido responsable el propio Tzetzes, hacia la que apuntaba Mastronarde $(\mathbf{Z})$; una tradición que, de haber existido, habría también tenido fuerte influencia en la génesis de $\gamma, \mathrm{y}$ hacia la que puede apuntar el Par. gr. 2707 (A Boiss.), que ya en 1300/1301 copia la versión más corta (sin añadidos marginales).

Con respecto al arquetipo $\boldsymbol{\alpha}^{\prime}$ - representado, por ejemplo, por el Vat. gr. 1369 (c Matr.) -, en él parece recuperarse la versión completa del poema, la que leíamos con el auxilio de versos marginales ya en $\mathbf{D}$ y $\mathbf{E}$, ambos de la segunda mitad del siglo XIII. Sin que se pueda excluir la influencia del arquetipo de $\boldsymbol{\alpha}$, la naturaleza misma del códice vaticano - con su prolijo aparato de marginalia y todo tipo de apuntes, en una estructura que denuncia la mano de un competente filólogo - hace posible que la inclusión de los versos en el cuerpo principal del poema haya sido labor del copista, interesado en la versión más completa posible, fusionada de entre las fuentes manuscritas de que disponía, la misma que presenta Matranga al editar los versos que faltan en Boissonade.

Pero la existencia de $\mathbf{Z}$ puede aún valerse de otro argumento, fácilmente detectable antes incluso de llevar a cabo una colación textual más exhaustiva. Hablamos de los versos 1-11 del canto XXIV del poema, solamente un ejemplo, entre muchos a lo largo del poema, de redacciones diferentes, para los cuales Matranga y Boissonade editan dos versiones completamente distintas:

\section{[Matr.]}

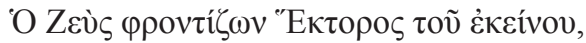

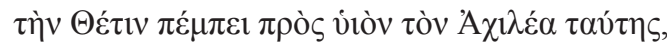

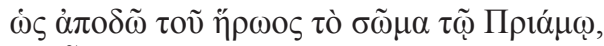

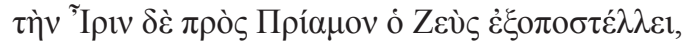

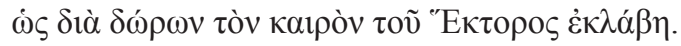

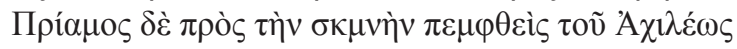

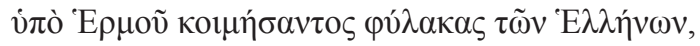

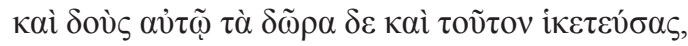

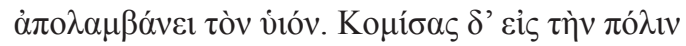

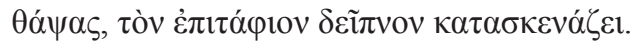

\section{[Boiss.]}

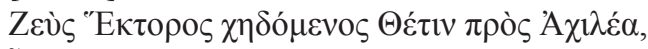

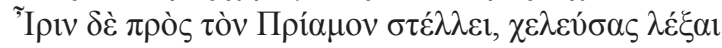

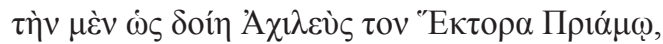

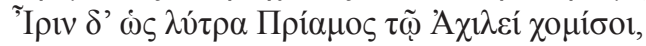

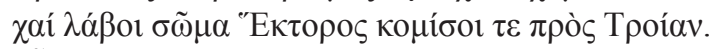

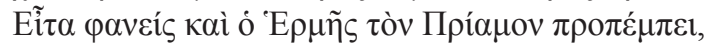

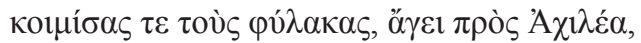

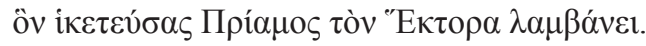

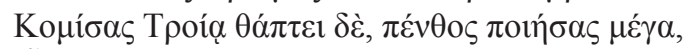

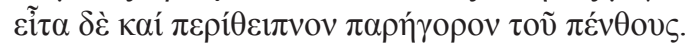


Los versos, parte del resumen del comienzo del canto XXIV de la Ilíada, tienen sentidos equivalentes en ambas versiones, aunque el léxico presente cambios muy significativos. La versión de Matranga es la que copian D y E, por lo que merece, como mínimo, ser considerada de igual valor textual que la que editó Boissonade, copiada de su códice C, el Par. gr. 2644, del siglo XIV (dichos versos faltan en los códices A y B de su edición, Par. Gr. 2707 y 2705 respectivamente, de la misma centuria). Admitiendo que ambas versiones corresponden a redacciones Tzetzianas - información que el tiempo permitirá confirmar o refutar -, y si volvemos a fijarnos en el stemma antes propuesto, la versión de Matranga parece tener relación con $\boldsymbol{\alpha}^{\prime}$ (y puede que con $\boldsymbol{\beta}$ ), no siendo imposible que la de Boissonade resulte de ese hipotético arquetipo $\gamma$. Una vez más, el Vat. gr. 1369 [c] sería ejemplo de códice dependiente de $\boldsymbol{\alpha}^{\prime}$, de esa fusión más tardía de versiones, en la medida en que, a la vez que copia los versos marginales ausentes de $\gamma$ en el cuerpo principal de texto, como antes se dijo, transmite el comienzo del canto XXIV como lo edita Boissonade (ff. $347 \mathrm{v}-348 \mathrm{r}$ ), quizás por influencia de la versión de $\boldsymbol{\beta}$.

Con el proceso de recensio apenas empezado, se resumen a continuación las conclusiones o hipótesis presentadas que justifican, desde luego, una nueva edición crítica de las Alegorías de la Ilíada: (1) Paleográficamente, jamás se ha colacionado la lección de al menos nueve copias completas del poema, de las cuales dos (D y E) deben de hecho constituir las fuentes textuales más antiguas, poco más de un siglo posteriores a la composición Tzetziana original. (2) La investigación preliminar que hemos podido realizar hasta el momento se inclina a dar razón a la sospecha de Mastronarde, de que el mismo Tzetzes haya revisado su obra y hecho circular al menos dos versiones de ella. De no ser así, parece seguro al menos que dicha bifurcación ha ocurrido muy temprano, como demostraría el hecho de que el manuscrito D (copiado entre 1250 y 1280, es decir, apenas cien años después de la composición del poema) ya incluye versos adicionales al margen. (3) El texto base para la nueva edición, que habría que enriquecer y precisar con las aportaciones de los manuscritos no colacionados, ha de ser, por tanto, el de Matranga (1850), que, a pesar de ser un año anterior al de Boissonade, edita bastantes más versos (algunos de ellos copiados al margen en los códices dependientes del arquetipo $\boldsymbol{\beta}$ ), de los cuales luego habría que juzgar la pertinencia de edición caso a caso.

Lo hasta aquí dicho puede sonar demasiado especulativo, pero no pretende ser más que una propuesta metodológica, por la cual nos ha parecido razonable empezar a clasificar los muchos testimonios manuscritos que deben ser tenidos en cuenta en una nueva edición de las Alegorías de la Ilíada, cuya necesidad es, tal vez, la única conclusión segura del trabajo hecho hasta ahora. Lo siguiente ha de ser la confirmación (o refutación) del stemma de transmisión primordial propuesto en este artículo, por vía del examen de todos los manuscritos identificados, lo que permitirá su división por familias y el establecimiento de los arquetipos que han de privilegiarse en la colación textual y la deseada (y sumamente necesaria) edición crítica del poema.

\section{Bibliografía}

Boissonade, J.Fr. (1851, reimpr. 1967), Tzetzae Allegoriae Iliadis. Accedunt Pselli Allegoriae quarum una inédita, Paris, Dumont. 
Braccini, T. (2011), «Riscrivere l'epica: Giovanni Tzetze di fronte al ciclo troiano», CentoPagine 5: 43-57.

Browning, R. (1975), «Homer in Byzantium», Viator 8: 15-33.

Browning, R. (1992), «The Byzantines and Homer», en R. Lamberton \& J.J. Keaney (eds.), Homer's Ancient Readers. The Hermeneutics of Greek Epic's Earliest Exegetes, Princeton, Princeton University Press: 134-148.

Budelmann, F. (2002), «Classical commentary in Byzantium: John Tzetzes on ancient Greek literature», en R.K. Gibson \& C.S. Kraus (eds.), The Classical Commentary. Histories, practices, theory, Leiden-Boston-Köln, Brill: 141-169.

Cesaretti, P. (1991), Allegoristi di Omero a Bisanzio: ricerche ermeneutiche, Milano, Guerini.

Coxe, H.O. (1883, reimpr. 1969), Catalogi codicum manuscriptorum Bibliothecae Bodleianae pars prima recensionem codicum Graecorum continens, IV.1, Oxford, University Press.

Goldwyn, A. J. \& Kokkini, D. (2015), John Tzetzes. Allegories of the Iliad, Cambridge (MA)London, Harvard University Press (Dumbarton Oaks Medieval Library, 37).

Goldwyn, A.J. (2017), «Theory and Method in John Tzetzes' Allegories of the Iliad and Allegories of the Odyssey», Scandinavian Journal of Byzantine and Modern Greek Studies 3: 141-171.

Hill, B. (1999), Imperial Women in Byzantium 1025-1204, New York, Taylor \& Francis Ltd. Hunger, H. (1955), «Johannes Tzetzes, Allegorien zur Odyssee, Buch XIII-XXIV», ByzZ 48: 11-38.

Hunger, H. (1956), «Johannes Tzetzes, Allegorien zur Odyssee I-XII», ByzZ 49: 249-310.

Hunger, H. (1961), Katalog der griechischen Handschriften der Österreichischen Nationalbibliothek, Teil 1: Codices Historici, Codices Philosophici et Philologici, Museion NF IV, 1, 1, Wien, Prachner.

Kaldellis, A. (2007), Hellenism in Byzantium. The Transformations of Greek Identity and the Reception of the Classical Tradition, Cambridge-New York, Cambridge University Press.

Kaldellis, A. (2009), «Classical Scholarship in Twelfth-Century Byzantium», en C. Barber \& D. Jenkins (coords.), Medieval Greek Commentaries on the Nicomachean Ethics, Leiden, Brill: 1-43.

Leone, P.L.M. (1995), Ioannis Tzetzae Carmina Iliaca, Catania, Cooperativa Universitaria Libraria Catanese.

Mastronarde, D.J. (2015), Rec. to Goldwyn \& Kokkini (2015), Bryn Mawr Classical Review 2015.09.45.

Matranga, P. (1850), Anecdota greca I e mss. bibliothecis Vaticana, Angelica, Barberiniana, Vallicelliana, Medicea, Vindobonensi deprompta, Roma, C.A. Bertinelli.

Matzukis, C. (1992), «Homer within the Byzantine framework», Akroterion 37.1: 2-5.

Morgan, G. (1983), «Homer in Byzantium: John Tzetzes». In C.A. Rubio \& C. W. Shelmerdine (eds.), Approaches to Homer, Austin, University of Texas Press: 165-168.

Rhoby, A. (2010), «Ioannes Tzetzes als Aufgtragsdichter», Graeco-Latina Brunensia 15.2: 155-170. 


\section{Láminas}

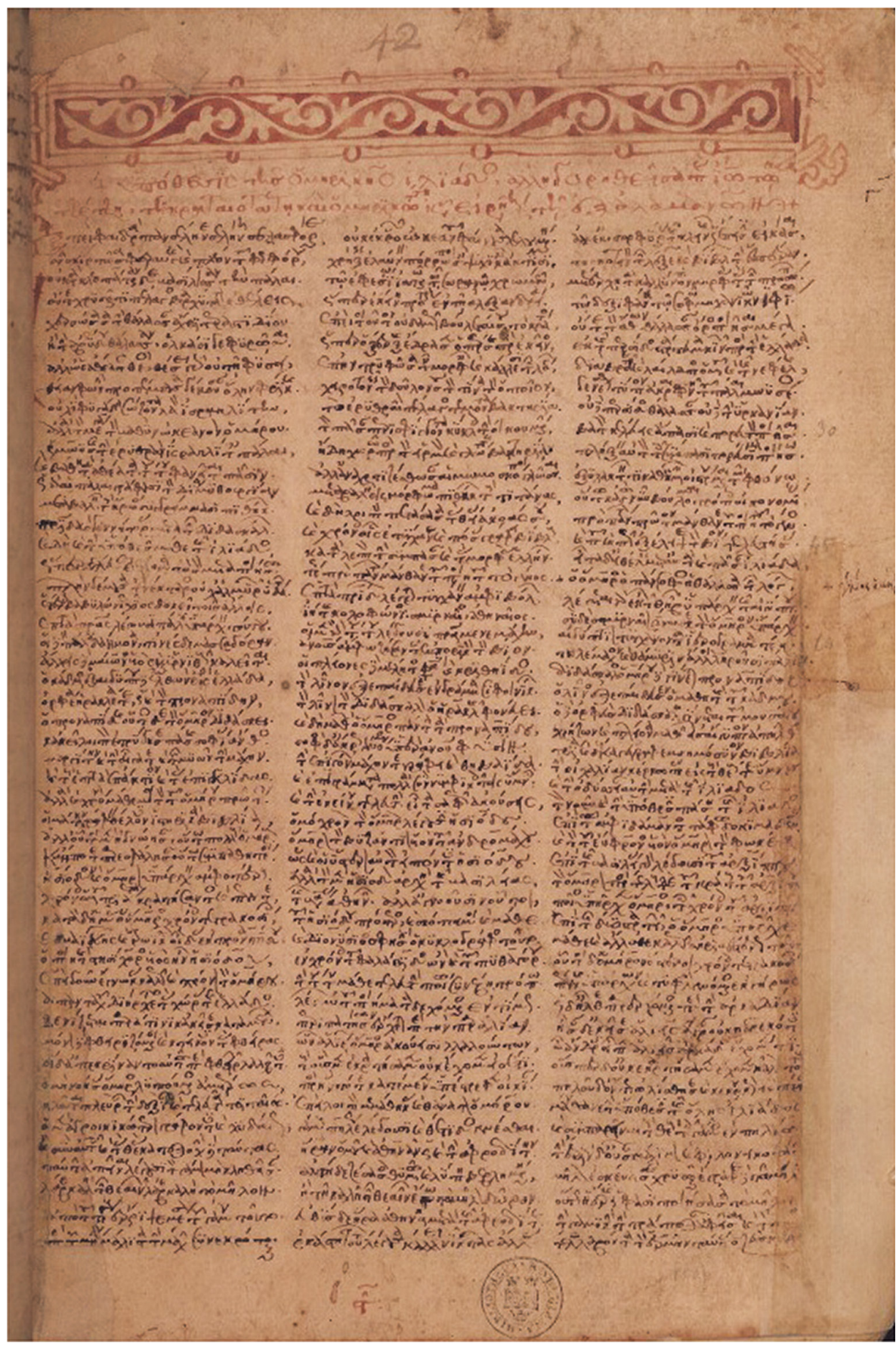

1. Barocci 131, fol. 42r. Biblioteca Bodleiana. Oxford. 1250-1280.

(C) Bodleian Libraries, University of Oxford. 


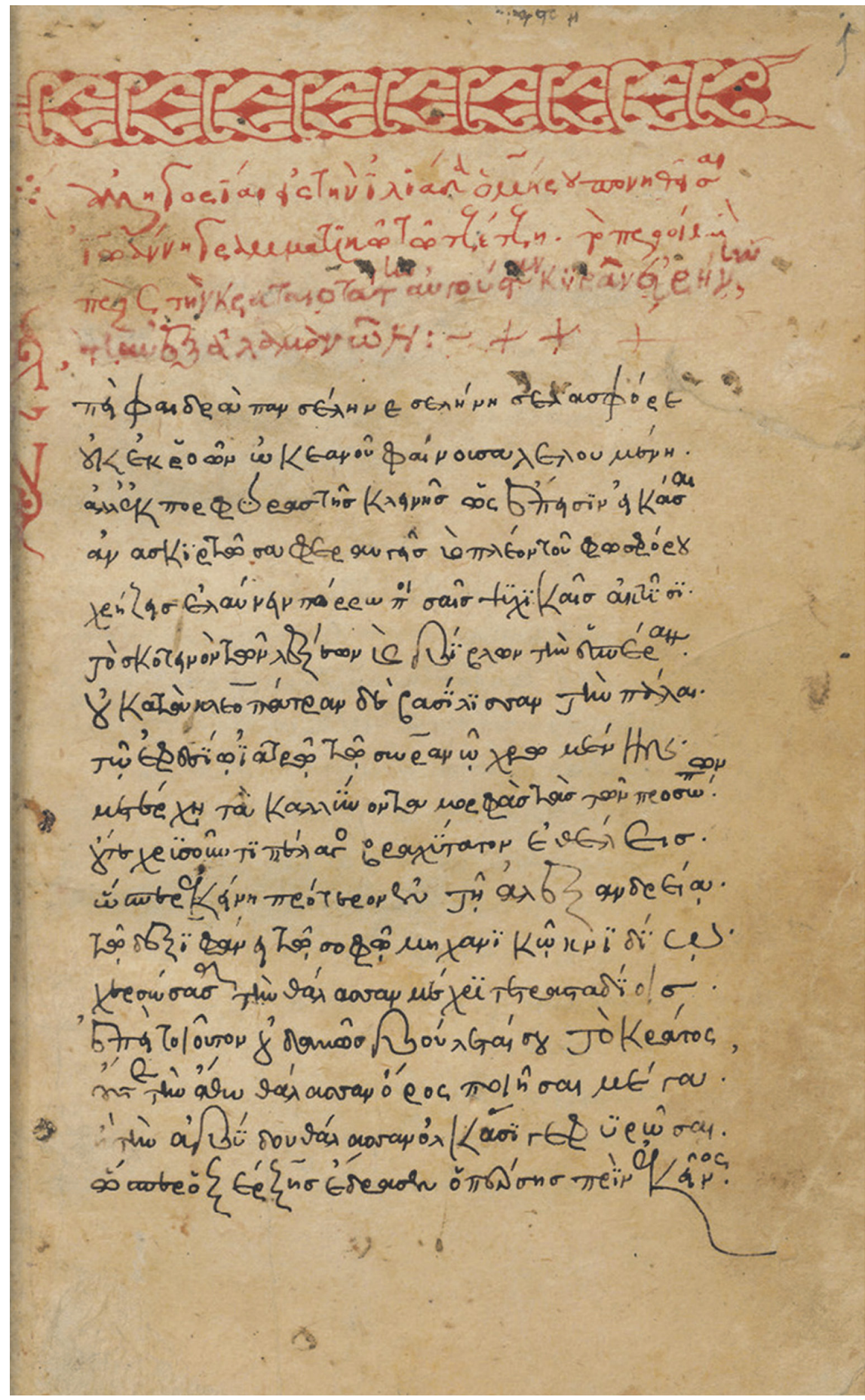

2. Vindob. phil. gr. 305, fol. 1r. Österreichische Nattionalbibliothek. 1280. (C) Österreichische Nattionalbibliothek, Sammlung von Handschriften und alten Drucken. 
